There may be a role for addition of rivaroxaban to aspirin in patients with stable coronary artery disease

\title{
Amitava Banerjee*
}

*Farr Institute of Health Informatics, University College London, London, UK

Correspondence to

Dr Amitava Banerjee, Farr Institute of Health Informatics, University College London, London WC1E 6BT, UK; ami.banerjee@ucl.ac.uk

Commentary on: Connolly SJ, Eikelboom JW, Bosch J, et al. Rivaroxaban with or without aspirin in patients with stable coronary artery disease: an international, randomised, double-blind, placebocontrolled trial. Lancet. 2017 S0140-6736:32458-3.

\section{Context}

Stable coronary artery disease (CAD), caused by both activation of platelets and the coagulation cascade, is a growing burden in all countries. - Compared with aspirin, combination therapy with warfarin and aspirin led to additional benefit against recurrent myocardial infarction and death. However, increased serious bleeding, including intracranial haemorrhage, has limited its use in clinical practice. ${ }^{2}$ To date, studies of the effects of direct anticoagulants, such as rivaroxaban (which inhibits factor $\mathrm{Xa}$ ), in stable CAD have conflicting results, and their role is still unclear. $\underline{34}$

\section{Methods}

An international (33 countries), double-blind, randomised, placebo-controlled trial recruited individuals with stable CAD in the outpatient setting. $\frac{5}{5}$ Eligibility criteria were myocardial infarction in the past 20 years, multivessel CAD, history of stable or unstable angina, previous multivessel percutaneous coronary intervention or previous multivessel coronary artery bypass graft surgery. Computer-generated randomisation after a 30-day run in period (1:1:1) was to rivaroxaban $(2.5 \mathrm{mg}$ orally twice daily) plus aspirin ( $100 \mathrm{mg}$ once daily), rivaroxaban alone (5 mg orally twice daily) or aspirin alone (100 mg orally once a day). Each treatment group was double dummy, with concealed treatment allocation for patients, study staff and researchers. The primary outcome was myocardial infarction, stroke or cardiovascular death.

\section{Findings}

A total of 24824 individuals had stable CAD. Follow-up had a mean duration of 1.95 years and was $99.8 \%$ complete. The study population had a mean age of 68.3 years (SD 7.8 ), which was $80 \%$ male and $69 \%$ had history of myocardial infarction. There were no significant differences in baseline characteristics between the three trial arms. Compared with aspirin alone, the primary outcome was reduced with rivaroxaban plus aspirin (347/8313 (4\%) vs 460/8261 (6\%); HR 0.74, 95\% Cl 0.65 to $0.86, p<0.0001)$ but not with rivaroxaban alone $(411 / 8250(5 \%)$ vs $460 / 8261(6 \%) ; \mathrm{HR} 0.89,95 \% \mathrm{Cl}$ 
0.78 to $1.02, \mathrm{p}=0.094)$. Compared with aspirin alone, combination therapy with rivaroxaban plus aspirin (263/8313 (3\%) vs $158 / 8261$ (2\%); HR $1.66,95 \% \mathrm{Cl} 1.37$ to $2.03, \mathrm{p}<0.0001$ ) and rivaroxaban alone (236/8250 (3\%) vs $158 / 8261$ (2\%); HR $1.51,95 \% \mathrm{Cl} 1.23$ to $1.84, \mathrm{p}<0.0001$ ) were associated with more major bleeds. Major bleeding was most commonly gastrointestinal: 130 (2\%) individuals on rivaroxaban plus aspirin, $84(1 \%)$ individuals on rivaroxaban alone and $61(1 \%)$ on aspirin alone. Compared with aspirin alone, rivaroxaban plus aspirin reduced mortality $(262 / 8313$ (3\%) vs $339 / 8261$ (4\%); HR 0.77, $95 \% \mathrm{Cl} 0.65$ to $0.90, p=0.0012$ ).

\section{Commentary}

This very large trial was multinational, well-designed, well-conducted and industry-sponsored. A combination of rivaroxaban and aspirin was superior to aspirin alone in reducing the composite outcome of myocardial infarction, stroke and cardiovascular mortality by $26 \%$. Rivaroxaban alone did not hold any advantage over aspirin alone for the primary outcome. Looking at the outcomes individually, there was a $23 \%$ reduction in mortality but no significant reduction in recurrent myocardial infarction. Addition of low-dose rivaroxaban to aspirin gave a consistent advantage, regardless of time after or presence of myocardial infarction. Compared with aspirin, major bleeds increased with combination therapy by $66 \%$ and with rivaroxaban alone by $51 \%$. Absolute risk reduction was only $2 \%$ for the primary composite endpoint. Therefore, the number needed to treat is 50 .

The trial population had very high adherence to other evidence-based therapies (eg, 92\% on lipidlowering agents, $72 \%$ on ACE inhibitor or angiotensin receptor blocker and $74 \%$ on beta-blocker), compared with real-world data.6

In summary, despite very low $\mathrm{p}$ values, there were small differences in absolute risk reduction. Even though fatal bleeding and intracerebral bleeding were not increased, net clinical benefit may not be in favour of the combination therapy with aspirin and rivaroxaban.

Implications for practice

There appears to be a role for addition of rivaroxaban to aspirin in patients with stable CAD, and the fact that the benefit was consistent whether myocardial infarction was recent or many years previously suggests that rivaroxaban may be useful over long periods of treatment. Rivaroxaban does not have a role as an alternative to aspirin in this context. However, the absolute risk reduction was small, and therefore, efforts may be better focused on improving adherence and persistence to well-established, existing evidence-based drugs, including aspirin, statins and angiotensin system blockers and beta-blockers.

\section{References}

1. Moran AE , Forouzanfar MH, Roth GA , et al . The global burden of ischemic heart disease in 1990 and 2010: the Global Burden of Disease 2010 study. Circulation 2014;129:1493501.doi:10.1161/CIRCULATIONAHA.113.004046

2. Anand SS , Yusuf S. Oral anticoagulants in patients with coronary artery disease. J Am Coll Cardiol 2003;41:S62-9.doi:10.1016/S0735-1097(02)02776-6

3. Mega JL, Braunwald E , Wiviott SD , et al . Rivaroxaban in patients with a recent acute coronary syndrome. N Engl J Med 2012;366:9-19.doi:10.1056/NEJMoa1112277

4. Alexander JH , Lopes RD, James $\mathrm{S}$, et al . Apixaban with antiplatelet therapy after acute coronary syndrome. N Engl J Med 2011;365:699-708.doi:10.1056/NEJMoa1105819 
5. Connolly SJ, Eikelboom JW , Bosch J, et al . Rivaroxaban with or without aspirin in patients with stable coronary artery disease: an international, randomised, double-blind, placebo-controlled trial. Lancet 2017. doi: 10.1016/S0140-6736(17)32458-3.

6. Yusuf S, Islam S, Chow CK, et al. Use of secondary prevention drugs for cardiovascular disease in the community in high-income, middle-income, and low-income countries (the PURE Study): a prospective epidemiological survey. Lancet 2011;378:1231-43.doi:10.1016/S0140-6736(11)61215-4 